\title{
Cerebral plasticity in acute vestibular deficit
}

\author{
Marco Alessandrini • Bianca Napolitano • \\ Ernesto Bruno • Letizia Belcastro • \\ Fabrizio Ottaviani $\cdot$ Orazio Schillaci
}

Received: 12 July 2008 / Accepted: 3 March 2009 / Published online: 18 March 2009

(C) Springer-Verlag 2009

\begin{abstract}
The aim of this study was to analyze the effect of acute vestibular deficit on the cerebral cortex and its correlation with clinical signs and symptoms. Eight righthanded patients affected by vestibular neuritis, a purely peripheral vestibular lesion, underwent two brain single photon emission computed tomography (SPECT) in 1 month. The first SPECT analysis revealed reduced blood flow in the temporal frontal area of the right hemisphere in seven of eight patients, independent of the right/left location of the lesion. The alteration was present always in the right, non-dominant hemisphere and was reversible in some patients 1 month after the onset, together with attenuation of signs and symptoms. It may be hypothesized that the transient reduction of cortical blood flow and subsequently of cortical activity in the non-dominant hemisphere, also the expression of cerebral plasticity, may serve as a defense mechanism aimed to attenuate the vertigo symptom.
\end{abstract}

Keywords Cerebral plasticity · Vestibular deficit . Cortical representation $\cdot$ SPECT

M. Alessandrini $\cdot$ B. Napolitano $\cdot$ E. Bruno $(\varangle)$.

L. Belcastro $\cdot$ F. Ottaviani

Department of Otolaryngology,

University of Rome "Tor Vergata",

Policlinico "Tor Vergata", Viale Oxford,

81, 00133 Rome, Italy

e-mail: bruno.ernesto@libero.it; bnapolitano@inwind.it

O. Schillaci

Department of Biopathology and Diagnostic Imaging,

University of Rome "Tor Vergata”, Rome, Italy

\section{Introduction}

For many years, research has concentrated on the labyrinth, brainstem and cerebellum to elucidate vestibular function. However, spatial orientation and perception of movement require processing of vestibular information at the cortical level and comparatively little is known about the cortical representation of the vestibular system.

Animal experiments have shown that there is no primary vestibular cortex that receives projections exclusively from vestibular afferents [1]. Instead, several multimodal sensory areas have been identified that integrate vestibular, visual and somato-sensory signals. In humans, these areas have been partly confirmed by intraoperative cortical stimulation [2], clinical studies in patients with acute cortical lesions [3] and functional imaging including blood flow measurements with single photon emission computed tomography (SPECT) [4, 5], functional magnetic resonance imaging (fMRI) [6-8] and positron emission tomography (PET) [9-11]. These latter studies aimed to identify a map of cortical areas activated during vestibular stimulation via caloric or galvanic stimuli.

A subsequent study analyzed the effect of a peripheral vestibular lesion on cerebral cortex processing of vestibular stimuli reporting metabolic changes in vestibular cortex during acute vestibular neuritis using a fluorodeoxyglucose positron emission tomography (FDG-PET) [12]. This study examined right-handed patients affected by right-sided vestibular neuritis.

The aim of the present study is to analyze the cortical representation of vestibular neuritis in right-handed subjects, including both right-sided and left-sided lesions. In particular, variations of cerebral blood flow have been measured by SPECT and correlated to clinical aspects of vestibular dysfunction. 
We selected patients affected by vestibular neuritis, a disease that we have already studied and described in previous papers [13]. Vestibular neuritis is also known as acute unilateral vestibular paralysis [14] and is the second most common cause of vertigo [15]. Although this disorder was first described at the beginning of the twentieth century [16], its pathogenesis is unknown [14, 17]. It tends to occur most frequently in 40-50-year olds, affects both sexes and all ethnic groups and is often associated with recent or concurrent upper respiratory infections [18]. Characteristic signs and symptoms of vestibular neuritis include: a sudden onset of severe rotational vertigo, sometimes remittent, which can be prolonged and associated with spontaneous nystagmus; a markedly reduced or totally absent caloric response in the affected ear; nausea; postural instability and no other neurologic or cochlear symptoms and findings $[14,18]$. Since vestibular neuritis is a purely peripheral lesion of the vestibular system, it constitutes an ideal "experimental model" of a partial vestibular de-afferentation, useful for observing the effect of vestibular de-afferentation on the cerebral cortex.

\section{Materials and methods}

Nine right-handed patients (male/female: 0.8; mean age: $51.6 \pm 13.84$ ) affected by vestibular neuritis constituted the study group. Every patient underwent a complete physical examination and blood analysis; an otoneurological evaluation including a thorough anamnesis, tonal liminar audiometry, assessment of spontaneous and evoked nystagmus by electronystagmographic recordings; a neurologic examination and a brain MRI.

All patients presented tonal liminar audiometry consistent with age and all clinical signs and symptoms associated with vestibular neuritis, such as sudden onset rotational vertigo persistent for hours, neurovegetative symptoms and the presence of spontaneous, persistent, horizontal nystagmus (II-III degree). Two patients had a vestibular neuritis on the right side and seven on the left side. All patients were observed within $48 \mathrm{~h}$ from the onset of symptoms. Brain MRI showed an absence of significant pathological signs in eight patients and a demyelinating disease in one patient, who was then excluded from the study.

The laterality quotient for right-handedness according to the 10-item inventory of the Edinburgh test was +100 in seven of the eight patients and +80 in the last patient [19].

Subjects underwent a brain technetium- $99 \mathrm{~m}$ SPECT within $72 \mathrm{~h}$ of the onset of symptoms and again after 1 month in subjects who had any abnormalities at the first SPECT exam.

Technetium-99 m ethyl cysteinate dimer (ECD) was prepared according to the instructions on the commercial vial (Neurolite, Bristol- Myers Squibb, MA, USA).
Brain SPECT was performed about 45 min after intravenous administration of $740 \mathrm{MBq}$ of ${ }^{99 \mathrm{~m}} \mathrm{Tc}-\mathrm{ECD}$, with the patient in a quiet, dark room. Dedicated physical restraints were used to immobilize the patient's head during data collection. SPECT images were acquired in a $128 \times 128$ matrix, using low energy, high resolution (LEHR) parallelhole collimators. The energy window was set at 140 $\operatorname{kev} \pm 10 \%$. Multiple views over $360^{\circ}$, with $30 \mathrm{~s}$ acquisition times per projection and an angular step of $3^{\circ}$ were obtained. Images were reconstructed using a Butterworth filtered back projection (cut off: 0.5 and order: 10). Transverse, sagittal, coronal slices were generated. Transaxial slices were oriented parallel to the canto-meatal line.

Images were visually evaluated by a well-experienced nuclear physician, masked to the results of any prior investigations. Normal SPECT findings consisted of homogeneous and symmetric tracer distribution in the gray matter of the cortex and basal ganglia. Abnormal SPECT findings were considered to be areas of hypoperfusion or asymmetry evident in at least two consecutive slices.

An otoneurological examination was repeated 1 month from the onset of symptoms with a complete re-evaluation of signs and symptoms. All subjects gave their informed consent to participate in the study, after the procedure and radiation risks had been explained.

\section{Results}

The first SPECT exam revealed reduced blood flow in the temporal frontal area of the right hemisphere in seven patients, independent of the side of the lesion (two patients were affected by right vestibular neuritis and five patients by left vestibular neuritis; Fig. 1). No alteration of cerebral blood flow was observed in one patient affected by left vestibular neuritis.

The second SPECT exam, performed after 1 month in the seven patients with reduced cerebral blood flow revealed normal blood flow in three patients and an improved blood flow in the other four patients (Table 1).

Thus, the alteration was always present in the right hemisphere and was reversible in some patients 1 month after onset. Clinical signs and symptoms at 1 month were characterized by the absence of rotational vertigo and absence/reduction of spontaneous nystagmus, but the presence of postural imbalance in all patients, regardless of changes in blood flow at that time.

\section{Discussion}

The vestibular, visual and somatosensory systems jointly perceive the spatial orientation of the human body and 


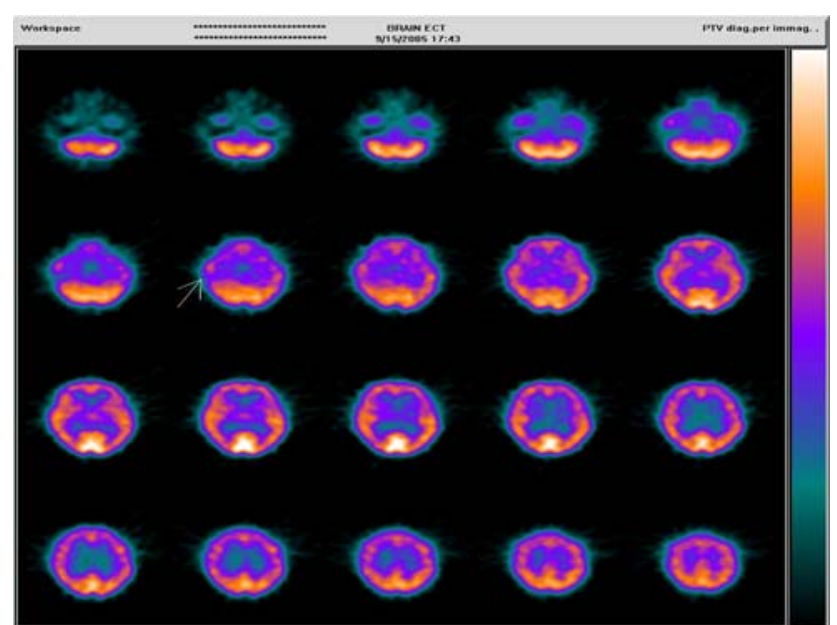

Fig. 1 99mTcECD Brain SPECT shows mild reduction of tracer uptake in the right fronto-temporal lobe (arrow)

coordinate the processes that play a role in bringing about this orientation. It is generally accepted that this function can be ascribed to a complex network of cortical and subcortical regions that continuously process afferent impulses, with one or another element of the network possibly predominating [20].

A number of animal experiments have been performed to identify elements of the network. As a result, areas with vestibular afferentation have been found in the parietal and temporal regions of primates $[1,2]$.

Parts of the network responsible for spatial orientation have been identified in patients with lesions of one cerebral hemisphere who suffer from contralateral spatial neglect syndrome [21]. Elements have been located in the inferior parietal lobe, the area of temporal parietal junction, the lateral region of the premotor cortex and certain subcortical regions such as the thalamus and basal ganglia [3, 21, 22].

The most widely used examination techniques in which stimulation is applied involve optokinetic, galvanic and caloric vestibular stimulation and the neck vibration test. Warm water caloric irrigation and cold gas caloric irrigation of the external auditory canal or galvanic vestibular nerve stimulation at the mastoid level cause bilateral activation of the temporoparietal cortex, especially the posterior insula and retroinsular region, which are the human homologs of the parietoinsular vestibular cortex (PIVC) in monkeys.

A human PET study during warm water caloric irrigation in right- and left-handed volunteers showed that two factors affect this bilateral cortical activation pattern of vestibular areas: the handedness of the subjects and the side of the stimulation. There is vestibular dominance in the non-dominant hemisphere and stronger activation occurs in the hemisphere ipsilateral to the stimulated ear [23]. A subsequent human PET study on right-handed patients affected by right-sided vestibular neuritis showed an activation of the primary vestibular cortex (parieto-insular vestibular cortex- PIVC) on the left side, contralateral to the affected side, and a deactivation of the supposed "secondary" multisensory vestibular cortex areas on the right side, ipsilateral to the affected ear [12]. The activation of "primary" vestibular cortex might be considered

Table 1 Population profile

\begin{tabular}{|c|c|c|c|c|}
\hline Patients & $\begin{array}{l}\text { Lesion } \\
\text { side }\end{array}$ & Brain MRI & SPECT $(24-72 \mathrm{~h})$ & SPECT (1 month follow-up) \\
\hline 1 & Right & Negative & $\begin{array}{l}\text { Reduced blood flow in the frontal } \\
\text { temporal lobe on the right side }\end{array}$ & Negative \\
\hline 2 & Right & Negative & $\begin{array}{l}\text { Reduced blood flow in the frontal } \\
\text { temporal lobe on the right side }\end{array}$ & $\begin{array}{l}\text { Attenuation of the reduced blood } \\
\text { flow in the frontal temporal } \\
\text { lobe on the right side }\end{array}$ \\
\hline 3 & Left & Negative & $\begin{array}{l}\text { Reduced blood flow in the } \\
\text { frontal temporal lobe on the right side }\end{array}$ & $\begin{array}{l}\text { Attenuation of the reduced blood } \\
\text { flow in the frontal temporal } \\
\text { lobe on the right side }\end{array}$ \\
\hline 4 & Left & Negative & $\begin{array}{l}\text { Reduced blood flow in the frontal } \\
\text { temporal lobe on the right side }\end{array}$ & Negative \\
\hline 5 & Left & Negative & $\begin{array}{c}\text { Reduced blood flow in the frontal } \\
\text { temporal lobe on the right side }\end{array}$ & $\begin{array}{l}\text { Attenuation of the reduced blood } \\
\text { flow in the frontal temporal } \\
\text { lobe on the right side }\end{array}$ \\
\hline 6 & Left & Negative & $\begin{array}{l}\text { Reduced blood flow in the frontal temporal } \\
\text { lobe on the right side }\end{array}$ & $\begin{array}{l}\text { Attenuation of the reduced } \\
\text { blood flow in the frontal } \\
\text { temporal lobe on the right side }\end{array}$ \\
\hline 7 & Left & Negative & $\begin{array}{l}\text { Reduced blood flow in the frontal } \\
\text { temporal lobe on the right side }\end{array}$ & Negative \\
\hline 8 & Left & Negative & Negative & \\
\hline 9 & Left & Demyelinating disease & & \\
\hline
\end{tabular}


the cortical correlate of the lesional tonic imbalance, whereas deactivation of the "secondary" multisensory vestibular cortex areas might reflect a cortical mechanism that suppresses the distressing consequences of erroneous perceptions of motion. However, this study analyzed only the right-sided lesions in right-handed patients and, referring to the reported results, the deactivation of secondary vestibular cortex on the right side might be considered as an ipsilateral deactivation as well as a deactivation of the non-dominant hemisphere.

The present study aimed to detect the cortical representation of vestibular neuritis in right-handed subjects, including both right-sided and left-sided lesions. Our results are not very accurate regarding identification and definition of distinct cerebral cortical areas involved in vestibular stimulation, because of the poor anatomical definition of the imaging technique used. Nevertheless, this information has been widely described in previous studies and we were interested in the hemispheric dominance of vestibular information processing.

Our results did show that all patients presented a reduced cerebral blood flow in distinct cortical areas situated in the right hemisphere, despite the side of the lesion. These data suggest that a strong vestibular stimulus, such as partial de-afferentation in case of vestibular neuritis, has its strongest effect in the non-dominant hemisphere. In fact, all the selected patients were right-handed and presented blood flow alteration in the right hemisphere.

Furthermore, this alteration of cerebral blood flow was completely reversible in three of seven patients and improved in four of seven patients about 1 month from the onset of symptoms, after symptoms and signs of vestibular de-afferentation had subsided. This suggests that the reduced blood flow was likely a result of the lesion stimulating the vestibular system.

Our results seem to confirm an asymmetric cortical activation pattern induced by unilateral peripheral vestibular loss as reported by Bense et al. [12]. In addition, we think that it would be interesting to demonstrate the cerebral activation pattern during acute vestibular deficit also with a more accurate technique other than SPECT. In fact, a deactivation of the right hemisphere in both right-sided and leftsided peripheral lesions, likely due to the dominance of vestibular function in the non-dominant hemisphere, might be considered as a defense mechanism aimed to attenuate the symptom vertigo.

This fact is confirmed by the clinical progress of the disease. After an early phase, characterized by intense vertiginous symptoms, the patients' symptoms decreased, despite the presence of spontaneous nystagmus, which is known to be a direct expression of the deafferentation. Although our data need confirmation with more accurate imaging techniques, it seems that the dominance of vestibular cortical function in the non-dominant hemisphere is more critical than the side of lesion and/or stimulation.

Conflict of interest The authors declare that they have no conflict of interest.

\section{References}

1. Grusser OJ, Pause M, Schreiter U (1990) Vestibular neurons in the parieto-insular cortex of monkeys (Macaca fascicularis): visual and neck receptor responses. J Physiol 430:559-583

2. Grusser OJ, Pause M, Schreiter U (1990) Localization and responses of neurons in the parieto-insular vestibular cortex of awake monkeys (Macaca fascicularis). J Physiol 430:537-557

3. Vallar G, Perani D (1986) The anatomy of unilateral neglect after right hemisphere stroke lesions: a clinical CT correlation study in man. Neuropsychologia 24:609-622

4. Friberg L, Olsen TS, Roland PE, Paulson OB, Lassen NA (1985) Focal increase of blood flow in the cerebral cortex of man during vestibular stimulation. Brain 108:609-623

5. Takeda N, Hashikawa K, Moriwaki H, Oku N, Koizuka I, Kitahara T et al (1996) Effects of caloric vestibular stimulation on parietal and temporal blood flow in human brain: a consecutive technetium-99-HMPAO SPECT study. J Vestib Res 6:127-134

6. Lobel E, Kleine JF, Le Bihan D, Leroy-Willig A, Berthoz A (1998) Functional MRI of galvanic vestibular stimulation. J Neurophysiol 80:2699-2709

7. Bense S, Stephan T, Yousry TA, Brandt T, Dieterich M (2001) Multisensory cortical signal increases and decreases during vestibular galvanic stimulation (fMRI). J Neurophysiol 85:886-899

8. Suzuki M, Kitano H, Ito R, Kitanishi T, Yazawa Y, Ogawa T et al (2001) Cortical and subcortical vestibular response to caloric stimulation detected by functional magnetic resonance imaging. Brain Res Cogn Brain Res 12:441-449

9. Bottini G, Sterzi R, Paulesu E, Vallar G, Cappa SF, Erminio F et al (1994) Identification of the central vestibular projections in man: a positron emission tomography activation study. Exp Brain Res 99:164-169

10. Bottini G, Karnath HO, Vallar G, Sterzi R, Frith CD, Frackowiak RSJ et al (2001) Cerebral representations for egocentric space: functional anatomical evidence from caloric vestibular stimulation and neck vibration. Brain 124:1182-1196

11. Wenzel R, Bartenstein P, Dieterich M, Danek A, Weindl A, Minoshima S et al (1996) Deactivation of human visual cortex during involuntary ocular oscillations: a PET activation study. Brain 119:101-110

12. Bense S, Bartenstein P, Lochmann M, Schlindwein P, Brandt T, Dieterich M (2004) Metabolic changes in vestibular and visual cortices in acute vestibular neuritis. Ann Neurol 56:624-630

13. Alessandrini M, D’Erme G, Bruno E, Napolitano B, Magrini A (2003) Vestibular compensation: analysis of postural rearrangement as a control index for unilateral vestibular deficit. Neuroreport 14:1-5

14. Strupp M, Arbusow V (2001) Acute vestibulopathy. Curr Opin Neurol 14:11-20

15. Dix MR, Hallpike CS (1952) The pathology, symptomatology and diagnosis of certain common disorders of the vestibular system. Ann Otol Rhinol Laryngol 61:987-1016

16. Ruttin B (1909) Zur differentialdiagnose der labyrinth-u. Horenrverkrankugen $Z$ Ohrenheilk 57:327-331

17. Strupp M, Brandt T (1999) Vestibular neuritis. Adv Otorhinolaryngol 55:111-136

18. Nadol JB Jr (1995) Vestibular neuritis. Otolaryngol Head Neck 112:162-172 
19. Oldfield RC (1971) The assessment and analysis of handedness: the Edinburgh inventory. Neuropsychologia 9:97-113

20. Guldin WO, Grusser OJ (1998) Is there a vestibular cortex? Trends Neurosci 21:254-259

21. Vallar G, Bottini G, Rusconi ML, Sterzi R (1993) Exploring somatosensory hemineglect by vestibular stimulation. Brain $116: 71-86$
22. Hussain M, Kennard C (1996) Visual neglect associated with frontal lobe infarction. J Neurol 243:652-657

23. Dieterich M, Bense S, Lutz S, Drzezga A, Stephan T, Bartenstein P et al (2003) Dominance for vestibular cortical function in the nondominant hemisphere. Cereb Cortex 13:994-1007 